\title{
ON THE FISK-TAIT EQUATION FOR SPIN-3/2 FERMIONS INTERACTING WITH AN EXTERNAL MAGNETIC FIELD IN NONCOMMUTATIVE SPACE-TIME
}

\author{
Ilyas HaouamiD \\ Laboratoire de Physique Mathématique et de Physique Subatomique (LPMPS), \\ Université Frères Mentouri, Constantine 25000, Algeria \\ (Received 27 March 2019; in final form 11 November 2019; accepted 27 November 2019; published online 18 February 2020)
}

\begin{abstract}
In this paper, we investigated the Fisk-Tait equation in interaction with an external magnetic field in noncommutative space-time. The space-time noncommutativity is introduced through the MoyalWeyl product method. Consequently, we studied the continuity equation in both commutative and noncommutative space-time; there we examined the influence of the space-time noncommutativity on the current density quadri-vector. Moreover, we find that the total charge obtained from the probability density is still indefinite even when space does not commute. Furthermore, we found the spin current density in the two different spin directions. We also investigated the linking between the fermions and the bosons in the Fock space using the Holstein-Primakoff transformation.

Key words: Fisk-Tait equation, spin-3/2 particles, noncommutative space-time, Moyal-Weyl product, noncommutative Fisk-Tait equation, continuity equation, spin-3/2 current density, Holstein-Primakoff transformation.
\end{abstract}

DOI: https://doi.org/10.30970/jps.24.1801

\section{INTRODUCTION}

Over the years, particle equations for an arbitrary spin were considered the subject for careful investigations. That is why today we are interested in the relativistic equations that describe the motion of spin-3/2 particles, such as the relativistic Rarita-Schwinger equation (1940) [1], the Fisk-Tait equation (1973) [2], Hurley's field equation [3], Bhabha-Gupta equation (Bhabha, Gupta $1952,1954,1974)[4,5]$, the approach for arbitrary spin equation by V. Bargman, E. P. Wigner (1948) [6]. Also the Heisenberg equations of motion for the spin- $3 / 2$ field (1977) [7] are applied to dynamic systems with constraints depending upon external fields, the Lagrange and Heisenberg equations of motion are the same for the quantized charged spin- $3 / 2$ field in the presence of a minimal external electromagnetic interaction. The RaritaSchwinger spin-3/2 equation in the weak-field limit is obtained to satisfy the Heisenberg equations of motion. This is similar to the case of spin- $3 / 2$ field minimally coupled with an external electromagnetic field by Mainland and Sudarshan (1973) [8].

As well recently, we have the link between the relativistic canonical quantum mechanics of arbitrary spin and the covariant local field theory by V. M. Simulik (2017) [9] (where the found equations are without redundant components). It has been confirmed that the synthesis of the relativistic canonical quantum mechanics of the spins-3/2 particle and antiparticle doublet is completely similar to the synthesis of the Dirac equation from the relativistic canonical quantum mechanics of the spin$1 / 2$ particle-antiparticle doublet. On the basis of the investigation of solutions and transformation properties with respect to the Poincaré group the obtained new 8-component equation is suggested to be well defined for the description of $\operatorname{spin} s=3 / 2$ fermions, unlike the Rarita-Schwinger equation which has 16 components and needs the additional condition.

The equation for particles of spin- $3 / 2$ originally was given by Fierz and Pauli (I939) in spinor form [10], while the Klein-Gordon, Dirac, and Proca equations provide a relativistic description of the particles that have the lowest spins cases $(S=0,1 / 2,1$ respectively).

For instance, the Rarita-Schwinger equation was formulated for the first time by William Rarita and Julian Schwinger, it was the most famous equation that describes the motion of the spin- $3 / 2$ fermions. However, in this research, we are interested in the Fisk-Tait equation. Because the Rarita-Schwinger equation and some of the mentioned equations at the top of the introduction contain many problems, such as the problem of causality, where the wavefront propagation described by the corresponding equation is greater and faster than that of the light [11], and also the problem of the imaginary energy. Unlike the mentioned equations, with FiskTait's equation all of these problems almost have been solved. To be clear, from the beginning all of the equations that describe the motion of spin- $3 / 2$ fermions have the problem of indefiniteness of the total charge [12, 13] including some exceptions such as in the case of the Gupta equation [14], were the charge density is positivedefinite for some properties, as well as in the theory of interacting spin-3/2 particle by T. Fukuyama and K. Yamamoto [15], where they suppress redundant particles by making their masses infinite. But these choices make the total charge in the free theory still indefinite.

Among the particles that have spin-3/2, we mention the gravitino, the quasi-spin- $3 / 2$ particles from the pairs of Cooper, and the baryon. This latter is a composite subatomic particle, which is a combination of at least three quarks such as $u$ (up quark), $d$ (down quark) and $s$ (strange quark) forming a baryon decuplet with spin- 
$3 / 2$, as in the case of baryons which are composed of one type of quark $(u u u, d d d, \ldots)$, these can exist in $J=3 / 2$ configuration. But in $J=1 / 2$ is forbidden by the Pauli exclusion principle. In the case of baryons which are composed of three types of quarks $(u d s, u d c, \ldots)$, these can exist in both $J=1 / 2$ and $J=3 / 2$ configurations. Taking into account that the first omega baryon discovered experimentally was the $\Omega^{-}$hyperon, made of three strange quarks, in 1964 [16], then recently, in 2017 the LHCb collaboration announced the observation of five new narrow $\Omega_{c}^{0}$ states decaying to $\Xi_{c}^{+} K^{-}$(an exceptionally large group of baryons) [17].

The baryons (strongly interacting fermions) are acted on by the strong nuclear force and are described by Fermi-Dirac statistics, which applies to all particles obeying the Pauli exclusion principle. Therefore, the two most studied groups of baryons are $S=1 / 2 ; L=0$ and $S=3 / 2 ; L=0$, which correspond to $J=1 / 2$ and $J=3 / 2$, respectively. Although they are not the only ones, it is also possible to obtain $J=3 / 2$ particles from $S=1 / 2$ and $L=2$, as well as $S=3 / 2$ and $L=2$ [18]. Theoretically, the baryons can have a higher spin of $3 / 2$.

The first known superconductor in which the quasispin-3/2 particles form Cooper pairs was created by American and New Zealand physicists [19]. The unconventional superconductor is an alloy of Yttrium, Platinum, and Bismuth, which is normally a topological semi-metal, the study was carried out by Johnpierre Paglione and his colleagues at the University of Maryland, Ames Laboratory of the Iowa State, Lawrence Berkeley national laboratory, and the universities of Otago and Wisconsin. In the alloy studied by Paglione and his colleagues, the charge is carried by quasiparticles of spin-3/2 particle type. These quasi-particles result from interactions between the electron spins and the positive charges of the atoms that compose the alloy; this influence is called the spin-orbit coupling and it is especially powerful in this material. The spin-3 state, which combines the moments of rotation and the orbital angular momentum, is the lowest energy state.

The gravitino $[20,21]$ is the superpartner of the graviton, predicted by the theories of the supergravity (this is a gauge fermion mediating supergravity interactions, and its existence is only a hypothesis). This spin- $3 / 2$ fermion obeys one of those equations mentioned at the top of the introduction, and in supersymmetry theory (SUSY) it is assumed that each fundamental particle has a superpartner. For a fermion (a particle with half-integer spin) such as the electron, its superpartner would be a particle with an integer spin, it is the selectron. For an integer spin particle such as the photon, its superpartner would be a half-integer particle called photino. For the quantum of gravitational force, in SUSY when the particle carrier is the graviton with spin-2, its superpartner is called the gravitino with spin-3/2; such theory is called the Gauged Super Gravity theory.

For many years, there has been interest in the study of spin- $3 / 2$ particles in as much detail as has been done for the spin- $1 / 2$ particles. So, our work can be considered a contribution that may help in the study of the spin- $3 / 2$ particles domain.

The goal of this work is to focus on the Fisk-Tait equation in a noncommutative space-time. Then we extract the continuity equations in both commutative and noncommutative space-time through the classical method, not using Lagrangian to extract the current density. Next, we study some additional contributions, such as the current density of the spin, also about how one can link the fermions with bosons in Fock space using the Holstein-Primakoff representation.

\section{THE SPIN-3/2 EQUATION IN AN EXTERNAL MAGNETIC FIELD IN NONCOMMUTATIVE SPACE-TIME}

\section{A. THE FISK-TAIT EQUATION}

For the concept of the product, we shall always sum over repeated indices. For example $A^{\mu} B_{\mu}=g^{\mu \nu} A_{\nu} B_{\mu}=$ $A_{0} B_{0}-\mathbf{A B}$, with Greek letters $\mu, \nu, \lambda$ being $0,1,2,3$ and $g^{\mu \nu}=\operatorname{diag}(1,-1,-1,-1)$ is the metric tensor in Minkowski space-time. We set $\hbar=c=1$ (natural units).

Recently, Fisk and Tait proposed an equation for spin-3/2 particles in 1973 [22], and they have shown that their equation remains causal even with minimal electromagnetic coupling, and made it free of other types of difficulties by which the Rarita-Schwinger formalism is plagued.

The wave function employed is a 24-component antisymmetric tensor-spinor $\Psi_{\lambda}^{\mu \nu}=-\Psi_{\lambda}^{\nu \mu}$. It transforms according to the following Lorentz group representation

$$
\begin{aligned}
& D\left(0, \frac{3}{2}\right) \oplus D\left(\frac{3}{2}, 0\right) \oplus D\left(\frac{1}{2}, 1\right) \\
& \oplus D\left(1, \frac{1}{2}\right) \oplus D\left(0, \frac{1}{2}\right) \oplus D\left(\frac{1}{2}, 0\right),
\end{aligned}
$$

with $\mu=\nu$ we have $\Psi^{\mu \mu}=0$, and the used wave function is as follows

$$
\Psi^{\mu \nu}=\left(\begin{array}{cccc}
0 & \Psi^{01} & \Psi^{02} & \Psi^{03} \\
-\Psi^{01} & 0 & \Psi^{12} & \Psi^{13} \\
-\Psi^{02} & -\Psi^{12} & 0 & \Psi^{23} \\
-\Psi^{03} & -\Psi^{13} & -\Psi^{23} & 0
\end{array}\right)
$$

With the quadri-vector of the electromagnetic field $A_{\mu}=\left(A_{0}, \mathbf{A}\right)$, the electromagnetic field tensor is defined as $F_{\mu \nu}=\partial_{\mu} A_{\nu}-\partial_{\nu} A_{\mu}$. In the presence of an external electromagnetic field, the wave function in commutative space-time obeys the following covariant equation [23]

$$
(\Gamma \pi+m B)_{\rho \sigma}^{\mu \nu} \Psi^{\rho \sigma}=0,
$$

with $\pi_{\lambda}=p_{\lambda}+e A_{\lambda}, p_{\lambda}=i\left(\partial / \partial x^{\lambda}\right)$ and the matrices $\left(\Gamma^{\lambda}\right)_{\rho \sigma}^{\mu \nu}$ and $B_{\rho \sigma}^{\mu \nu}$ are given by

$$
B_{\rho \sigma}^{\mu \nu}=g_{\rho}^{\mu} g_{\sigma}^{\nu},
$$

$$
\begin{aligned}
& \left(\Gamma^{\lambda}\right)_{\rho \sigma}^{\mu \nu}=-\frac{4}{3} \gamma^{\lambda} g_{\rho}^{\mu} g_{\sigma}^{\nu}-\frac{1}{3} \gamma^{\lambda}\left(\gamma^{\mu} \gamma_{\sigma} g_{\rho}^{\nu}-\gamma^{\nu} \gamma_{\sigma} g_{\rho}^{\mu}\right) \\
& +\frac{1}{3}\left(\gamma^{\mu} g_{\rho}^{\lambda} g_{\sigma}^{\nu}-\gamma^{\nu} g_{\rho}^{\lambda} g_{\sigma}^{\mu}-g^{\lambda \mu} \gamma_{\rho} g_{\sigma}^{\nu}+g^{\lambda \nu} \gamma_{\rho} g_{\sigma}^{\mu}\right) .(5)
\end{aligned}
$$


The gamma matrices obey the following Clifford covariant algebra

$$
\gamma^{\mu} \gamma^{\nu}+\gamma^{\nu} \gamma^{\mu}=2 g^{\mu \nu}, \sigma^{\mu \nu}=\frac{1}{2} i\left(\gamma^{\mu} \gamma^{\nu}-\gamma^{\nu} \gamma^{\mu}\right)
$$

where $\gamma^{0}=\left(\begin{array}{cc}1 & 0 \\ 0 & -1\end{array}\right), \gamma^{5}=\gamma^{0} \gamma^{1} \gamma^{2} \gamma^{3}, \quad \gamma=$ $\left(\begin{array}{cc}0 & \boldsymbol{\sigma} \\ -\boldsymbol{\sigma} & 0\end{array}\right)$. Note that $\gamma^{0}$ is Hermitian, and $\gamma$ is antiHermitian.

Using Eqs.(4, 5), the Fisk-Tait equation in a more detailed form is given by

$$
m \Psi^{\mu \nu}-\frac{4}{3} \gamma^{\lambda} \pi_{\lambda} \Psi^{\mu \nu}+\frac{1}{3} \gamma^{\lambda} \pi_{\lambda}\left(\gamma^{\nu} \gamma_{\sigma} \Psi^{\mu \sigma}-\gamma^{\mu} \gamma_{\sigma} \Psi^{\nu \sigma}\right)+\frac{1}{3}\left(\gamma^{\mu} \pi_{\rho} \Psi^{\rho \nu}-\gamma^{\nu} \pi_{\rho} \Psi^{\rho \mu}-\gamma_{\rho} \pi^{\mu} \Psi^{\rho \nu}+\gamma_{\rho} \pi^{\nu} \Psi^{\rho \mu}\right)=0
$$

The employed wave function tensor-spinor is decomposed in two wave vectors components as

$$
\begin{aligned}
& \Psi^{\mu \nu}=\left(\begin{array}{l}
\psi \\
\chi
\end{array}\right), \\
& \psi=\left(\Psi^{01}, \Psi^{02}, \Psi^{03}\right), \quad \chi=\left(\Psi^{23}, \Psi^{31}, \Psi^{12}\right) .
\end{aligned}
$$

The spin-3/2 parts of the wave function are

$$
\psi_{3 / 2}=\psi+\frac{1}{3} \gamma(\gamma \psi), \chi_{3 / 2}=\chi+\frac{1}{3} \gamma(\gamma \chi),
$$

with the subsidiary conditions $\gamma \psi_{3 / 2}=\gamma \chi_{3 / 2}=0$.

In case of a constant real magnetic field, we have the following expression

$$
F_{\mu \nu}=\left(\begin{array}{cccc}
0 & 0 & 0 & 0 \\
0 & 0 & \mathcal{H} & 0 \\
0 & -\mathcal{H} & 0 & 0 \\
0 & 0 & 0 & 0
\end{array}\right)
$$

where $\mathcal{H}$ is a constant real magnetic field oriented along the axis $(\mathrm{Oz})$. This present physical system is often referred to as the Landau system. For this problem, a convenient choice of the electromagnetic field vector is the following symmetric gauge

$$
\mathbf{A}=\frac{\mathcal{H}}{2}(-y, x, 0), \quad \text { with } A_{0}=0
$$

For simplicity, we may choose Landau gauge to reduce A to one component such that either

$$
\mathbf{A}=\mathcal{H}(0, x, 0) \quad \text { or } \quad \mathbf{A}=\mathcal{H}(-y, 0,0)
$$

It should be stressed that the expression of $\mathbf{A}$ in the symmetric and Landau gauges is obtained by using Eq. (10). Therefore, the Fisk-Tait equation (3) goes to

$$
\left(\Gamma^{\lambda}\right)_{\rho \sigma}^{\mu \nu} p_{\lambda} \Psi^{\rho \sigma}-e A_{k}\left(\Gamma^{k}\right)_{\rho \sigma}^{\mu \nu} \Psi^{\rho \sigma}+m B_{\rho \sigma}^{\mu \nu} \Psi^{\rho \sigma}=0, k=1,2,3
$$

with

$$
\left(\Gamma^{k}\right)_{\rho \sigma}^{\mu \nu}=-\frac{4}{3} \gamma^{k} g_{\rho}^{\mu} g_{\sigma}^{\nu}-\frac{1}{3} \gamma^{k}\left(\gamma^{\mu} \gamma_{\sigma} g_{\rho}^{\nu}-\gamma^{\nu} \gamma_{\sigma} g_{\rho}^{\mu}\right)+\frac{1}{3}\left(\gamma^{\mu} g_{\rho}^{k} g_{\sigma}^{\nu}-\gamma^{\nu} g_{\rho}^{k} g_{\sigma}^{\mu}-g^{k \mu} \gamma_{\rho} g_{\sigma}^{\nu}+g^{k \nu} \gamma_{\rho} g_{\sigma}^{\mu}\right)
$$

In the general case of a non-constant magnetic field, we introduce a function depending on $x$ in the Landau gauge as $A_{2}=x \mathcal{H} f(x)$ which gives us a non-constant magnetic field. The magnetic field can be calculated easily using $\mathcal{H}=\nabla \times \mathbf{A}$ which gives [24]

$$
\mathcal{H}(x)=\left(x \mathcal{H} \frac{d}{d x} f(x)+\mathcal{H} f(x)\right) \mathbf{e}_{3} .
$$

The above equation represents a special kind of nonconstant magnetic fields. If we specify $f(x)$, we obtain different classes of the non-constant magnetic field. If we take $f(x)=1$, in this case we get a constant magnetic field. Let us consider $f(x)=\frac{1}{x}\left(1-e^{-x}\right)$ to obtain an exponentially decaying magnetic field. Of course, a multi- tude of other possibilities exists, so that Eq. (15) goes to

$$
\mathcal{H}(x)=\left(\mathcal{H} e^{-x}\right) \mathbf{e}_{3}
$$

\section{B. THE FISK-TAIT EQUATION IN NONCOMMUTATIVE SPACE-TIME}

The noncommutative geometry [25-27] is the theory in which space may not commute anymore, so that one deals with a $4 \mathrm{~d}$ noncommutative space-time (NCST). The usual space-time coordinates $x^{\mu}$ are replaced by the operators $\hat{x}^{\mu}$ which satisfy the commutation relations [28]

$$
\left[\hat{x}^{\mu}, \hat{x}^{\nu}\right]=i \Theta^{\mu \nu}, \quad(\mu \nu=0, \ldots, 3),
$$


where the noncommutativity parameter is a real-valued antisymmetric constant matrix with a dimension of $\left[\right.$ length $\left.^{2}\right]$. And the uncertainty relations $\Delta \hat{x}^{\mu} \Delta \hat{x}^{\nu} \geq$ $\left|\Theta^{\mu \nu}\right| / 2$ are compatible with Eq. (17). For some studies about noncommutative systems concerning the noncommutative parameter, there are some experiments that have linked the noncommutative parameter scale to $\Theta \approx 4.10^{-40} \mathrm{~m}^{2}$ (which corresponds to $\Theta \approx 10^{-2} \mathrm{TeV}^{-2}$ ). This value corresponds to the upper bound on the fundamental coordinate scale [29], this bound will be automatically canceled when the magnetic field used in the experiment is weak $B \approx 5 \mathrm{mG}$. For our needs, in the context of the field theory, it is more practical to introduce the noncommutative scale $\Lambda_{\mathrm{nc}}$ by [30]

$$
\Theta^{\mu \nu}=\frac{c^{\mu \nu}}{\Lambda_{\mathrm{nc}}},
$$

were $c^{\mu \nu}$ is a dimensionless antisymmetric tensor, where the components are $\mathcal{O}(1) . \Lambda_{\text {nc }}$ represents a characteristic energy scale for the noncommutative theory which is necessarily quite large.

We would like to note that, it would be quite well to approach the problem via the Seiberg-Witten maps if we have an electromagnetic field interaction. But while we have a magnetic field interaction, we will follow a quite standard approach that has been widely used in the literature on noncommutative quantum mechanics (NCQM); which depends on obtaining a noncommutative version of a given field theory, and is based on replacing the product of the fields by the Moyal-Weyl product

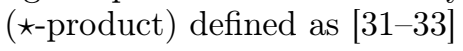

$$
\begin{aligned}
(f \star g)(x) & =\left.\exp \left[\frac{i}{2} \Theta_{\mu \nu} \partial_{x_{\mu}} \partial_{x_{\nu}}\right] f\left(x_{\mu}\right) g\left(x_{\nu}\right)\right|_{x_{\mu}=x_{\nu}} \\
& =f(x) g(x)+\sum_{n=1}\left(\frac{1}{n !}\right)\left(\frac{i}{2}\right)^{n} \Theta^{\mu_{1} \nu_{1}} \ldots \Theta^{\mu_{n} \nu_{n}} \partial_{\mu_{1}} \ldots \partial_{\mu_{k}} f(x) \partial_{\nu_{1}} \ldots \partial_{\nu_{k}} g(x)
\end{aligned}
$$

We want to emphasize that the non-commutative field theories for the low energies $\left(\Theta E^{2}<1\right)$ or the slowly varying fields reduce to their commutative version $(\Theta=$ $0)$ due to the nature of the Moyal $\star$-product.

It is well known that the noncommutative coordinates operator $\hat{x}^{\mu}$ is linked to the commutative one through the Heisenberg-Weyl algebra in terms of the following Darboux transformation

$$
\hat{x}^{\mu}=x^{\mu}-\frac{\Theta^{\mu \nu}}{2} p_{\nu}, \hat{p}^{\mu}=p^{\mu} .
$$

The variables $x^{\mu}, p^{\mu}$ satisfy the usual canonical commutation relations in QM. In what follows, we study the dynamic equation in NCST for the case of constant and non-constant magnetic fields using the Landau gauge. The Fisk-Tait equation in NCST is written as follows

$$
\begin{aligned}
& \left\{\left(\Gamma^{\lambda}\right)_{\rho \sigma}^{\mu \nu} p_{\lambda}+m B_{\rho \sigma}^{\mu \nu}\right\} \Psi^{\rho \sigma} \\
& +e\left(\Gamma^{\lambda}\right)_{\rho \sigma}^{\mu \nu} A_{\lambda}(x) \star \Psi^{\rho \sigma}(x)=0,
\end{aligned}
$$

where the $\star$-product is a realization of algebra (19). One can write the noncommutative part as

$$
\begin{aligned}
& \left(\Gamma^{\lambda}\right)_{\rho \sigma}^{\mu \nu} A_{\lambda}(x) \star \Psi^{\rho \sigma}(x) \approx\left(\Gamma^{\lambda}\right)_{\rho \sigma}^{\mu \nu} A_{\lambda} \Psi^{\rho \sigma}+\frac{i}{2} \Theta^{\alpha \beta}\left(\Gamma^{\lambda}\right)_{\rho \sigma}^{\mu \nu} \partial_{\alpha}\left\{A_{\lambda}\right\} \partial_{\beta} \Psi^{\rho \sigma}-\frac{1}{8}\left(\Theta^{\alpha \beta}\right)^{2}\left(\partial_{\alpha}\right)^{2}\left\{\left(\Gamma^{\lambda}\right)_{\rho \sigma}^{\mu \nu} A_{\lambda}\right\}\left(\partial_{\beta}\right)^{2} \Psi^{\rho \sigma} \\
& +\ldots \sum_{n=3}\left(\frac{1}{n !}\right)\left(\frac{i}{2}\right)^{n} \Theta^{a_{1} b_{1}} \ldots \Theta^{a_{n} b_{n}} \partial_{a_{1}} \ldots \partial_{a_{k}}\left\{\left(\Gamma^{\lambda}\right)_{\rho \sigma}^{\mu \nu} A_{\lambda}\right\} \partial_{b_{1}} \ldots \partial_{b_{k}} \Psi^{\rho \sigma} .
\end{aligned}
$$

However, one must pay attention to the ordering issues that can arise. In addition, with the help of Eq. (20), one can check that the expression (22) can be rewritten as
To the first order of $\Theta$, taking into account the case of potential $A_{2}=\mathcal{H} x$, the derivations in algebra (22) roughly stop in the first order of $\Theta$, then the approxi- 
mation gives

$$
\begin{aligned}
& \left\{\left(\Gamma^{\lambda}\right)_{\rho \sigma}^{\mu \nu} p_{\lambda}+m B_{\rho \sigma}^{\mu \nu}\right\} \Psi^{\rho \sigma}-e \mathcal{H} x\left(\Gamma^{2}\right)_{\rho \sigma}^{\mu \nu} \Psi^{\rho \sigma} \\
& -\frac{i e}{2} \mathcal{H} \Theta^{\alpha \beta}\left(\Gamma^{2}\right)_{\rho \sigma}^{\mu \nu} \partial_{\alpha}\{x\} \partial_{\beta} \Psi^{\rho \sigma}+0\left(\Theta^{2}\right)=0 .
\end{aligned}
$$

We can see that to this order, our noncommutative Fisk-Tait equation suggests the following quantity as a noncommutative correction

$$
\begin{aligned}
& \frac{i e}{2} \mathcal{H} \Theta^{\alpha \beta}\left(\Gamma^{2}\right)_{\rho \sigma}^{\mu \nu}\{x\} \partial_{\beta} \Psi^{\rho \sigma} \\
& =\frac{i e}{2} \mathcal{H} \Theta^{1 \beta}\left(\Gamma^{2}\right)_{\rho \sigma}^{\mu \nu} \partial_{\beta} \Psi^{\rho \sigma},
\end{aligned}
$$

the most important feature of this approximation is its simple character, which allows one to work simply to test the noncommutativity effect on the total charge through the continuity equation later, without having to worry about the issues coming from the arising series of the *-product.

\section{CASE OF A NON-CONSTANT MAGNETIC FIELD}

Using Eq. (12) and Eq. (16) would lead to a nonconstant magnetic field $A_{2}=\mathcal{H} x e^{-x}$, which provides a noncommutative Fisk-Tait equation for all orders of $\Theta$, as follows

$$
\left\{\left(\Gamma^{\lambda}\right)_{\rho \sigma}^{\mu \nu} \pi_{\lambda}+m B_{\rho \sigma}^{\mu \nu}\right\} \Psi^{\rho \sigma}-e\left(\Gamma^{2}\right)_{\rho \sigma}^{\mu \nu} \sum_{n=1}\left(\frac{1}{n !}\right)\left(\frac{i}{2}\right)^{n} \Theta^{a_{1} b_{1}} \ldots \Theta^{a_{n} b_{n}} \partial_{a_{1}} \ldots \partial_{a_{k}}\left\{\mathcal{H} x e^{-x}\right\} \partial_{b_{1}} \ldots \partial_{b_{k}} \Psi^{\rho \sigma}=0
$$

below we discuss some cases of the noncommutative correction part:

To first order of $\Theta(n=1)$, the approximation yields

$$
\mathcal{C}_{1}=\frac{i}{2} \Theta^{\alpha \beta}\left(\Gamma^{2}\right)_{\rho \sigma}^{\mu \nu} \partial_{\alpha}\left\{\mathcal{H} x e^{-x}\right\} \partial_{\beta} \Psi^{\rho \sigma}=\frac{i}{2} \mathcal{H} \Theta^{1 \beta}\left(\Gamma^{2}\right)_{\rho \sigma}^{\mu \nu}(1-x) e^{-x} \partial_{\beta} \Psi^{\rho \sigma} .
$$

To second order of $\Theta(n=2)$, with $a_{1}=a_{2}=1, b_{1}=b_{2}=\beta$, the approximation yields

$$
\mathcal{C}_{2}=\mathcal{C}_{1}+\frac{1}{8} \mathcal{H}\left(\Theta^{1 \beta}\right)^{2}\left(\Gamma^{\lambda}\right)_{\rho \sigma}^{\mu \nu}(2-x) e^{-x}\left(\partial_{\beta}\right)^{2} \Psi^{\rho \sigma} .
$$

To third order of $\Theta(n=3)$, with $a_{1}=a_{2}=a_{3}=1, b_{1}=b_{2}=b_{3}=\beta$, the approximation yields

$$
\mathcal{C}_{3}=\mathcal{C}_{2}+\frac{i}{48} \mathcal{H}\left(\Theta^{1 \beta}\right)^{3}\left(\Gamma^{\lambda}\right)_{\rho \sigma}^{\mu \nu}(3-x) e^{-x}\left(\partial_{\beta}\right)^{3} \Psi^{\rho \sigma}
$$

To calculate the $n^{\text {th }}$ order term, we consider that, $a_{1}=$ $a_{2}=\ldots=a_{n}=1, b_{1}=b_{2}=\ldots=b_{n}=\beta$, we have

$$
\begin{aligned}
\mathcal{C}_{n} & =\mathcal{C}_{n-1}-\frac{1}{n !}\left(\frac{i}{2}\right)^{n} \\
& \times\left(\Theta^{1 \beta}\right)^{n}\left(\Gamma^{\lambda}\right)_{\rho \sigma}^{\mu \nu}(n-x) e^{-x}\left(\partial_{\beta}\right)^{n} \Psi^{\rho \sigma} .
\end{aligned}
$$

From the comparison between commutative and noncommutative systems, it is widely obvious that the $\star-$ product causes noncommutative corrections in the FiskTait equation due to the presence of the magnetic field. These correction are controlled by the form and type of the magnetic field. It can be shown that, in NCST, if we take a constant magnetic field, the order of the noncommutativity cannot be higher than the first order. On the other hand, when we take a non-constant magnetic field (at least to the form of the non-constant magnetic field we have considered), it causes high orders in noncommutativity, which produce an infinite series of $\Theta$.

In the noncommutative Fisk-Tait equation, the appearance of terms proportional to explicit $\Theta$ parameter, in fact, is due to the noncommutativity effect on the dynamical equation. In the case of a constant magnetic field, we consider the appeared correction term as a kind of potential. Therefore, we will see whether the total charge obtained from the probability density remains indefinite or no.

It should also be noted that the case of higher dimensions of $\Theta$ and a more general algebra are complicated, all along with our physical systems involving both electrical and magnetic potentials.

\section{THE CONTINUITY EQUATION IN TWO TYPES OF SPACE-TIME}

\section{A. THE CONTINUITY EQUATION IN COMMUTATIVE CASE}

The Fisk-Tait equation in the commutative space-time is given by Eq. (7), with the following subsidiary conditions

$$
\gamma_{\mu} \gamma_{\nu} \Psi^{\mu \nu}=0, \pi_{\mu} \gamma_{\nu} \Psi^{\mu \nu}=0
$$


The complex conjugate of Eq. (7) is

$$
\begin{aligned}
& m \Psi^{\dagger \mu \nu}-\frac{4}{3} \Psi^{\dagger \mu \nu} \pi_{\lambda}^{*} \gamma^{\lambda \dagger}+\frac{1}{3}\left(-\Psi^{\dagger \nu \sigma} \gamma_{\sigma}^{\dagger} \gamma^{\dagger \mu} \gamma^{\dagger \lambda}+\Psi^{\dagger \mu \sigma} \gamma_{\sigma}^{\dagger} \gamma^{\dagger \nu} \gamma^{\dagger \lambda}\right) \pi_{\lambda}^{*} \\
& +\frac{1}{3}\left(\Psi^{\dagger \rho \nu} \pi_{\rho}^{*} \gamma^{\dagger \mu}-\Psi^{\dagger \rho \mu} \pi_{\rho}^{*} \gamma^{\dagger \nu}-\Psi^{\dagger \rho \nu} \pi^{* \mu} \gamma_{\rho}^{\dagger}+\Psi^{\dagger \rho \mu} \pi^{* \nu} \gamma_{\rho}^{\dagger}\right)=0 .
\end{aligned}
$$

Here $*$ and $\dagger$ stand for the complex conjugation of $\pi$ (even the potentials) and the wave functions, tensors respectively.

By multiplying $\Psi_{\mu \nu}^{\dagger}$ by Eq. (7), we have

$$
\begin{aligned}
& m \Psi_{\mu \nu}^{\dagger} \Psi^{\mu \nu}-\frac{4}{3} \Psi_{\mu \nu}^{\dagger} \gamma^{\lambda} \pi_{\lambda} \Psi^{\mu \nu}+\frac{1}{3} \Psi_{\mu \nu}^{\dagger} \gamma^{\lambda} \pi_{\lambda}\left(-\gamma^{\mu} \gamma_{\sigma} \Psi^{\nu \sigma}+\gamma^{\nu} \gamma_{\sigma} \Psi^{\mu \sigma}\right) \\
& +\frac{1}{3}\left(\Psi_{\mu \nu}^{\dagger} \gamma^{\mu} \pi_{\rho} \Psi^{\rho \nu}-\Psi_{\mu \nu}^{\dagger} \gamma^{\nu} \pi_{\rho} \Psi^{\rho \mu}-\Psi_{\mu \nu}^{\dagger} \gamma_{\rho} \pi^{\mu} \Psi^{\rho \nu}+\Psi_{\mu \nu}^{\dagger} \gamma_{\rho} \pi^{\nu} \Psi^{\rho \mu}\right)=0 .
\end{aligned}
$$

Multiplying Eq. (32) by $\Psi_{\mu \nu}$, we obtain

$$
\begin{aligned}
& m \Psi^{\dagger \mu \nu} \Psi_{\mu \nu}-\frac{4}{3} \Psi^{\dagger \mu \nu} \pi_{\lambda}^{*} \gamma^{\lambda \dagger} \Psi_{\mu \nu}+\frac{1}{3}\left(-\Psi^{\dagger \nu \sigma} \gamma_{\sigma}^{\dagger} \gamma^{\dagger \mu} \gamma^{\dagger \lambda}+\Psi^{\dagger \mu \sigma} \gamma_{\sigma}^{\dagger} \gamma^{\dagger \nu} \gamma^{\dagger \lambda}\right) \pi_{\lambda}^{*} \Psi_{\mu \nu} \\
& +\frac{1}{3}\left(\Psi^{\dagger \rho \nu} \pi_{\rho}^{*} \gamma^{\dagger \mu} \Psi_{\mu \nu}-\Psi^{\dagger \rho \mu} \pi_{\rho}^{*} \gamma^{\dagger \nu} \Psi_{\mu \nu}-\Psi^{\dagger \rho \nu} \pi^{* \mu} \gamma_{\rho}^{\dagger} \Psi_{\mu \nu}+\Psi^{\dagger \rho \mu} \pi^{* \nu} \gamma_{\rho}^{\dagger} \Psi_{\mu \nu}\right)=0 .
\end{aligned}
$$

With some minor simplifications and by subtracting Eq. (33) from Eq. (34), and by taking $\pi_{\lambda}=i \partial_{\lambda}$ for simplicity, which means considering the Fisk-Tait equation without the electromagnetic interaction, we find

$$
\partial_{\lambda} \mathcal{J}^{\lambda}=\partial_{\lambda}\left(-\frac{4}{3} \Psi^{\dagger \mu \nu} \gamma^{\lambda} \Psi^{\mu \nu}-\frac{2}{3} \Psi^{\dagger \mu \nu} \gamma^{\lambda} \gamma^{\mu} \gamma_{\sigma} \Psi^{\nu \sigma}+\frac{2}{3} \Psi^{\dagger \mu \nu} \gamma^{\mu} \Psi^{\lambda \nu}-\frac{2}{3} \Psi^{\dagger \lambda \nu} \gamma_{\rho} \Psi^{\rho \nu}\right)=0 .
$$

The above equation is the continuity equation, where the current density quadri-vector $\mathcal{J}^{\lambda}=\left(\mathcal{J}^{0}, \mathcal{J}^{i}\right)$ is

$$
\mathcal{J}^{\lambda}=-\frac{2}{3}\left(2 \bar{\Psi}^{\mu \nu} \gamma^{\lambda} \Psi_{\mu \nu}+\bar{\Psi}^{\mu \nu} \gamma^{\lambda} \gamma_{\mu} \gamma^{\sigma} \Psi_{\nu \sigma}-\bar{\Psi}^{\mu \nu} \gamma_{\mu} \Psi_{\nu}^{\lambda}+\bar{\Psi}^{\lambda \nu} \gamma^{\rho} \Psi_{\rho \nu}\right), \quad \bar{\Psi}^{\mu \nu}=\Psi^{\dagger \mu \nu} \gamma^{0} .
$$

The corresponding conserved quantity is the total probability

$$
\mathcal{J}^{0}=-\frac{2}{3}\left(2 \Psi^{\dagger \mu \nu} \Psi_{\mu \nu}+\Psi^{\dagger \mu \nu} \gamma_{\mu} \gamma^{\sigma} \Psi_{\nu \sigma}-\Psi^{\dagger \mu \nu} \gamma^{0} \gamma_{\mu} \Psi_{\nu}^{0}+\Psi^{\dagger 0 \nu} \gamma^{0} \gamma^{\rho} \Psi_{\rho \nu}\right)
$$

where $\mathcal{J}^{0}$ is the probability density for finding a particle at a particular position, and $\mathcal{J}^{i}$ is the current density of the spin-3/2 particle. It is clear that the total charge $\mathcal{Q}$ obtained from the probability density is indefinite

$$
\mathcal{Q}=\int \mathcal{J}^{0} d^{3} x=\int d^{3} x\left(-\frac{4}{3} \Psi^{\dagger i j} \Psi^{i j}+2 \Psi^{\dagger 0 i} \Psi^{0 i}-\frac{2}{3} \Psi^{\dagger i j} \gamma_{i} \gamma^{k} \Psi_{j k}+\frac{2}{3} \Psi^{\dagger o i} \gamma_{i} \gamma^{j} \Psi_{k i}\right)
$$

Using Eqs. $(8,9)$, we obtain

$$
\mathcal{Q}=2 \int d^{3} x\left(\psi_{3 / 2}^{\dagger} \psi_{3 / 2}-\chi_{3 / 2}^{\dagger} \chi_{3 / 2}\right)
$$

For all the spin- $3 / 2$ equations of motion, the problem of total charge indefiniteness remained [12], while in the case of Gupta equation [14] with some conditions, the charge density is positive-definite. However, generally the total charge in the free theory is indefinite, which means that the condition for the positivity of the charge is first noted, then it is shown that this condition has to be violated if the causality of propagation is to be ensured. The lack of a positive definite probability density suggests that many of the problems that plague the attempts to quantize spin-3/2 fields are also to be found in the classical field equations.

It should be noted, when considering the Fisk-Tait equation in interaction with EMF (which means taking $\pi_{\lambda}=p_{\lambda}+e A_{\lambda}$ ), that the calculations of the continuity equation lead to getting a correction term (of probability density type) in the continuity equation and the total charge expressions. We are going to show this in the next subsection together with the noncommutativity influence. 


\section{B. THE CONTINUITY EQUATION IN NONCOMMUTATIVE CASE}

The Fisk-Tait equation in NCPS is given by Eq. (24), and its complex conjugate is given by

$$
\begin{aligned}
& m \Psi^{\dagger \mu \nu}-\frac{4}{3} \Psi^{\dagger \mu \nu} p_{\lambda}^{*} \gamma^{\lambda \dagger}+\frac{1}{3}\left(-\Psi^{\dagger \nu \sigma} \gamma_{\sigma}^{\dagger} \gamma^{\dagger \mu} \gamma^{\dagger \lambda}+\Psi^{\dagger \mu \sigma} \gamma_{\sigma}^{\dagger} \gamma^{\dagger \nu} \gamma^{\dagger \lambda}\right) p_{\lambda}^{*} \\
& +\frac{1}{3}\left(\Psi^{\dagger \rho \nu} p_{\rho}^{*} \gamma^{\dagger \mu}-\Psi^{\dagger \rho \mu} p_{\rho}^{*} \gamma^{\dagger \nu}-\Psi^{\dagger \rho \nu} p^{* \mu} \gamma_{\rho}^{\dagger}+\Psi^{\dagger \rho \mu} p^{* \nu} \gamma_{\rho}^{\dagger}\right) \\
& -e \mathcal{H} x \Psi^{\dagger \rho \sigma}\left(\Gamma^{\dagger 2}\right)_{\rho \sigma}^{\mu \nu}+\frac{i e}{2} \mathcal{H} \Theta^{1 \beta}\left(\partial_{\beta} \Psi^{\rho \sigma}\right)^{\dagger}\left(\Gamma^{2 \dagger}\right)_{\rho \sigma}^{\mu \nu}=0 .
\end{aligned}
$$

Note that it is enough to consider the simplest case concerning the magnetic field $A_{2}=\mathcal{H} x$ to study the total charge within the noncommutativity in an explicitly solvable model. With $p_{\lambda}=i \partial_{\lambda}$, we multiply $\Psi_{\mu \nu}^{\dagger}$ by Eq. (24) and Eq. (40) by $\Psi_{\mu \nu}$. Therefore, by subtracting $\Psi_{\mu \nu}^{\dagger}$ Eq. (24) from Eq. (40) $\Psi_{\mu \nu}$, we obtain

$$
\begin{aligned}
& i \partial_{\lambda}\left[-\frac{4}{3} \bar{\Psi}^{\mu \nu} \gamma^{\lambda} \Psi_{\mu \nu}-\frac{2}{3} \bar{\Psi}^{\mu \nu} \gamma^{\lambda} \gamma_{\mu} \gamma_{\sigma} \Psi_{\nu \sigma}+\frac{2}{3} \bar{\Psi}^{\mu \nu} \gamma_{\mu} \Psi_{\nu}^{\lambda}-\frac{2}{3} \bar{\Psi}^{\lambda \nu} \gamma^{\rho} \Psi_{\rho \nu}\right] \\
& +e \mathcal{H} x\left[\Psi^{\dagger \rho \sigma}\left(\Gamma^{\dagger 2}\right)_{\rho \sigma}^{\mu \nu} \Psi_{\mu \nu}-\Psi_{\mu \nu}^{\dagger}\left(\Gamma^{2}\right)_{\rho \sigma}^{\mu \nu} \Psi^{\rho \sigma}\right] \\
& -\frac{i e}{2} \mathcal{H} \Theta^{1 \beta}\left[\Psi_{\mu \nu}^{\dagger}\left(\Gamma^{2}\right)_{\rho \sigma}^{\mu \nu} \partial_{\beta} \Psi^{\rho \sigma}+\Psi^{\dagger \rho \sigma} \partial_{\beta}\left(\Gamma^{\dagger 2}\right)_{\rho \sigma}^{\mu \nu} \Psi_{\mu \nu}\right]=0 .
\end{aligned}
$$

We contract the above equation as follows

$$
\partial_{\lambda} \mathcal{J}^{\lambda}+\zeta^{2}+\zeta_{\text {nc }}^{2}=0 .
$$

Eq. (42) will be recognized as the noncommutative continuity equation; we denote the separate terms in it as follows

$$
\begin{aligned}
\mathcal{J}^{\lambda} & =-\frac{4}{3} \bar{\Psi}^{\mu \nu} \gamma^{\lambda} \Psi_{\mu \nu}-\frac{2}{3} \bar{\Psi}^{\mu \nu} \gamma^{\lambda} \gamma_{\mu} \gamma_{\sigma} \Psi_{\nu \sigma}+\frac{2}{3} \bar{\Psi}^{\mu \nu} \gamma_{\mu} \Psi_{\nu}^{\lambda}-\frac{2}{3} \bar{\Psi}^{\lambda \nu} \gamma^{\rho} \Psi_{\rho \nu} \\
\zeta^{2} & =e \mathcal{H} x\left[\Psi^{\dagger \rho \sigma}\left(\Gamma^{\dagger 2}\right)_{\rho \sigma}^{\mu \nu} \Psi_{\mu \nu}-\Psi_{\mu \nu}^{\dagger}\left(\Gamma^{2}\right)_{\rho \sigma}^{\mu \nu} \Psi^{\rho \sigma}\right] \\
\zeta_{\mathrm{nc}}^{2} & =\frac{-e}{2} \mathcal{H} \Theta^{1 \beta}\left[\Psi_{\mu \nu}^{\dagger}\left(\Gamma^{2}\right)_{\rho \sigma}^{\mu \nu} \partial_{\beta} \Psi^{\rho \sigma}+\Psi^{\dagger \rho \sigma} \partial_{\beta}\left(\Gamma^{\dagger 2}\right)_{\rho \sigma}^{\mu \nu} \Psi_{\mu \nu}\right] .
\end{aligned}
$$

$\mathcal{J}^{\lambda}$ is the current density quadri-vector of the spin$3 / 2$ particle. The quantity $\zeta^{2}$ is a term of the probability density type; this quantity is related to the effect of the electromagnetic field interaction on the FiskTait equation. This quantity emerged merely as a term containing the constant magnetic field contribution, consequently after extracting the commutative or noncommutative continuity equation, this contribution being responsible for generating this probability density quantity. The correction term $\zeta_{\text {nc }}^{2}$ is a new noncommutative quantity of the current density type, the existence of this quantity corresponds to the explicit space noncommutative parameter which is involved in the obtained noncommutative continuity equation, that is because of the influence of the space noncommutativity on the spin- $3 / 2$ particle motion equation. And such quantity appeared as a term which is proportional to the noncommutativity parameter $\Theta$, then after extracting the noncommutative continuity equation, this term was responsible for generating the new quantity term of the current density type. Once the magnetic field is null, the quantities $\zeta^{2}$ and $\zeta_{\mathrm{nc}}^{2}$ will disappear.

We see that the total charge $\mathcal{Q}$ obtained from the probability density $\mathcal{J}^{0}$ still is indefinite even when space does not commute. To demonstrate its indefiniteness, it is enough to consider the rest frame in which $p=0$. Then Eq. (31) reduces to

$$
\gamma_{i} \gamma_{j} \Psi^{i j}=0, \quad \gamma_{i} \Psi^{0 i}=0,
$$

so that

$$
\mathcal{Q}^{\mathrm{nc}}=\mathcal{Q}+\zeta^{2}+\zeta_{\mathrm{nc}}^{2}=\int \mathcal{J}^{0} d^{3} x+\zeta^{2}+\zeta_{\mathrm{nc}}^{2} .
$$

It is easily seen that relations (44) do not eliminate the indefiniteness of $\mathcal{Q}^{\text {nc }}$.

\section{AN ADDITIONAL CONTRIBUTION}

There are several ideas from electricity, magnetism, and nuclear physics that suggest the contribution of the 
spin and the current density. In this section, we try to highlight some of those ideas. We have $S=\left(S_{1}, S_{2}, S_{3}\right)$; knowing that $S_{1}, S_{2}, S_{3}$ have a block diagonal form, and while $S=3 / 2, \operatorname{dim}[S]=2\left(\frac{3}{2}\right)+1=4$, this leads to obtaining the following spin-eigenvalues

$$
-3 / 2 \leq m_{S} \leq+3 / 2,
$$

which means to have

$$
m_{S}=-\frac{3}{2},-\frac{1}{2}, \frac{1}{2}, \frac{3}{2},
$$

with $S_{ \pm}=S_{1} \pm i S_{2},\left[S_{1}, i S_{2}\right]=2 S_{3}$ and $\left\{S^{2}, S_{3}, S_{ \pm}\right\}$is a complete set of commuting observables (CSCO), and according to our representation (1), we restrict ourselves only to the following eigenvectors

$$
\left|0, \frac{3}{2}\right\rangle,\left|\frac{3}{2}, 0\right\rangle,\left|\frac{1}{2}, 1\right\rangle,\left|1, \frac{1}{2}\right\rangle,\left|0, \frac{1}{2}\right\rangle,\left|0, \frac{1}{2}\right\rangle .
$$

\section{A. THE SPIN CURRENT DENSITY}

We are interested in the spin-eigenvalue $m_{S}=\frac{3}{2}$, and from Eq. (8) our wave function with spin is

$$
\begin{aligned}
& \Psi^{\mu \nu}(x, t)=\left(\begin{array}{c}
\psi\left(x,+\frac{3}{2}, t\right) \\
\chi\left(x,-\frac{3}{2}, t\right)
\end{array}\right)=\left(\begin{array}{c}
\psi_{\uparrow} \\
\chi_{\downarrow}
\end{array}\right), \\
& \Psi^{\dagger \mu \nu}=\left(\psi^{*}\left(x,+\frac{3}{2}, t\right) \chi^{*}\left(x,-\frac{3}{2}, t\right)\right)=\left(\begin{array}{ll}
\psi_{\uparrow}^{*} & \chi_{\downarrow}^{*}
\end{array}\right) .
\end{aligned}
$$

This suggests that the probability density function is used to find the spin-3/2 particle (at a point $x$ and a moment $t$ ) and also to determine the current density, in which it is composed of the parts of the two different spin directions.

The orbital motion of the spin- $3 / 2$ particle causes the current density, where the spin causes a magnetic moment, in which it can be expressed by its corresponding current. Besides, we term this part of the current density as $\mathcal{J}_{S}$ (spin current density), and this cannot occur in the continuity equation because of the indefiniteness of the total charge.

We determine the spin current density using Maxwell's equation, where

$$
\operatorname{rot} \mathbf{B}=4 \pi(\mathbf{J}+\operatorname{rot}\langle\mathbf{M}\rangle) .
$$

We have to replace the magnetization $\langle\mathbf{M}\rangle$ by the average density of the magnetic moment, the up-direction part of the magnetic dipole is given by

$$
\left\langle\mathbf{M}_{\uparrow}\right\rangle=-\mu \psi_{\uparrow}^{*} \mathbf{S} \psi_{\uparrow},
$$

with

$$
\mathbf{S}=\frac{1}{2} \sum_{\alpha, \beta=1,2,3} c_{\alpha}^{\dagger} \boldsymbol{\sigma}_{\alpha \beta} c_{\beta}
$$

$\boldsymbol{\sigma}_{1}, \boldsymbol{\sigma}_{2}, \boldsymbol{\sigma}_{3}$ are the usual Pauli matrices, $c_{i}^{\dagger}, c_{i}$ are creation and annihilation operators, $\widehat{n}_{i}=c_{i}^{\dagger} c_{i}$ is the number operator of spin-3/2 particles at the site $i$. And the intrinsic magnetic moment $\mu=\frac{q}{3 m} \mathbf{S}$ [34], which relates the magnetic moment to the spin angular momentum, that corresponds to the gyromagnetic ratio $\frac{q}{3 m}$; the highest eigenvalue of the operator $\mu$ is given by

$$
\mu_{\max }=\frac{|q|}{2 m} .
$$

Using the above properties, Eq. (50) becomes

$$
\operatorname{rot} \mathbf{B}_{\uparrow}=4 \pi\left(\mathbf{J}_{\uparrow}-\mu \operatorname{rot}\left(\psi_{\uparrow}^{*} \mathbf{S} \psi_{\uparrow}\right)\right),
$$

with

$$
\mathbf{J}_{\uparrow S}=-\mu \operatorname{rot}\left(\psi_{\uparrow}^{*} \mathbf{S} \psi_{\uparrow}\right),
$$

knowing that the spin current density is composed of the parts of the two different spin directions. For another direction, the spin current density is

$$
\mathbf{J}_{\downarrow S}=-\mu \operatorname{rot}\left(\chi_{\downarrow}^{*} \mathbf{S} \chi_{\downarrow}\right),
$$

We note that for the up-direction wave function components, we have only $\left\{\Psi^{01}, \Psi^{02}, \Psi^{03}\right\}$, but for the down-direction wave function components, we have $\left\{\Psi^{23}, \Psi^{31}, \Psi^{12}\right\}$.

Knowing that the obtained spin current density cannot be affected by the noncommutativity in space-time, unlike the considered Maxwell's equation, it would be influenced by the noncommutativity in space-time as

$$
\operatorname{rot} \mathbf{B}_{\uparrow \downarrow}=4 \pi\left(\mathbf{J}_{\uparrow \downarrow}+\mathcal{J}_{n c \uparrow \downarrow}^{2}+\operatorname{rot}\left\langle\mathbf{M}_{\uparrow \downarrow}\right\rangle\right) .
$$

\section{B. THE HOLSTEIN-PRIMAKOFF TRANSFORMATION}

As mentioned in the introduction, the graviton with spin-2 in SUSY would have a superpartner called the gravitino with spin-3/2. We want to show how to connect the spin operators from boson with fermions spin operators in Fock space using the HolsteinPrimakoff $(\mathcal{H P})$ transformation [35]; the idea here is to connect a system of $2 N+1$ fermions onto a system of $N$ bosons. This calculation can be found in books, and it has been considered originally by C. E. Alonso and his colleagues in 1992 [36]. The spin and angular momentum eigenfunctions are labeled with the quantum numbers $n, m, l, j \ldots$

The fermion space is defined in terms of fermion creation (annihilation) operators $c_{i j}^{+}\left(c_{i j}\right)$. The boson-fermion space is defined in terms of boson creation (annihilation) operator $B_{i j}^{+}\left(B_{i j}\right)$, with new fermion creation (annihilation) operators $a_{i j}^{+}\left(a_{i j}\right)$, knowing that the boson and the fermion operators commutate with each other. With $\left[B_{i j}, B_{\alpha \beta}^{+}\right]=\delta_{i \alpha} \delta_{j \beta}-\delta_{i \beta} \delta_{j \alpha},\left[B_{i j}, B_{\alpha \beta}\right]=0$, the HolsteinPrimakoff image of the single-fermion creation operator $c_{s_{1} m_{1}}^{+}$is given by [37]

$$
\left(c_{s_{1} m_{1}}^{+}\right)_{\mathcal{H} \mathcal{P}}=\sum_{s_{2} m_{2}}\left\{\mathcal{T}_{s_{1} m_{1} s_{2} m_{2}} a_{s_{2} m_{2}}^{+}+B_{s_{1} m_{1} s_{2} m_{2}}^{+} a_{s_{2} m_{2}}\right\}
$$


with the operator $\mathcal{T}=\sqrt{I-B^{+} B}$, that characterizes the $\mathcal{H} \mathcal{P}$ boson expansion and is to be interpreted by its Taylor series expansion, $\left(c_{i j}^{+}\right)_{\mathcal{H P}}$ denotes the HolsteinPrimakoff image of $c_{i j}^{+}$.

The first-order of the expansion of $\mathcal{T}$ (square-root function) is obtained

$$
\mathcal{T}_{s_{1} m_{1} s_{2} m_{2}}=\delta_{s_{1} s_{2}} \delta_{m_{1} m_{2}}-\frac{1}{2}\left(B^{+} B\right)_{s_{1} m_{1} s_{2} m_{2}}
$$

In fact, the expansion of $\mathcal{T}$ (with $B^{+} B \ll 1$ ) gives us $\sum_{i=0}^{\infty} a_{i}\left(B^{+} B\right)^{i}$. The Taylor series for the operator $\mathcal{T}$ is expected to converge very slowly, so that its development is likely to require many terms, since high order terms in the series translate into many boson operators. Physically the utility of such approach is questionable, so that J. Dukelsky and S. Pittel [38] proposed to accelerate the convergence of the series by choosing a better initial value for $\left(B^{+} B\right)_{0}$. This leads to Eq. (59), where

$$
\left(B^{+} B\right)_{s_{1} m_{1} s_{2} m_{2}}=\sum_{s_{3} l_{1} l_{2} \alpha \beta} l_{1} l_{2} \alpha(-1)^{s_{3}+m_{1}+l_{2}+\alpha+\beta} X_{j_{2} j_{3}}^{L_{1}} X_{j_{1} j_{3}}^{L_{2}}\left(\begin{array}{ccc}
s_{1} & s_{2} & \alpha \\
m_{1} & -m_{2} & \beta
\end{array}\right) \times\left(\begin{array}{ccc}
l_{1} & l_{2} & \alpha \\
s_{1} & s_{2} & \beta
\end{array}\right)\left(\gamma_{L_{1}}^{+} \times \tilde{\gamma}_{L_{2}}\right)_{\beta}^{\alpha}
$$

We can determine the expression of the boson spin components in the Fock space

$$
\begin{aligned}
& S_{3}=S-n_{b}, \\
& S^{+}=\sqrt{2 s-n_{b}} b, \\
& S^{-}=b^{+} \sqrt{2 s-n_{b}},
\end{aligned}
$$

with $n_{b}=b^{+} b=1,2,3, \ldots$ Knowing that $J=\sqrt{2 j+1}$, which describes the interaction between spins, $b$ is a boson operator, says Holstein-Primakoff. The operator $\gamma_{b}^{+}$creates the collective boson of multipolarity $b$, which is given by

$$
\gamma_{\alpha \beta}^{+}=\frac{1}{2} \sum_{j_{1} j_{2}} X_{s_{1} s_{2}}^{\alpha} B_{s_{1} s_{2} \alpha \beta}
$$

and $X_{s_{1} s_{2}}^{a}$ are the structure coefficients of $\gamma_{a}^{+}, B_{s_{1} s_{2} \alpha \beta}$ are the angular momenta coupled to boson creation operators.

To link the quadrupole operator using the $\mathcal{H} \mathcal{P}$ expansion, it is usually possible to directly use the image of the particle-hole fermion operator, which is given by

$$
\left(c_{i}^{+} c_{j}\right)_{H P}=\sum_{k} B_{i k}^{+} B_{j k}+a_{i}^{+} a_{j}
$$

Non-physical states appear for $n_{b} \geq 2 S$, as under these conditions, the spin projection on direction 3 is greater than $S$, so that the use of this spin representation strongly has a meaning for the low temperature and for large spin values ( $n_{b}$ is very weak).

\section{CONCLUSION}

In conclusion, the space-time noncommutativity is introduced in the Fisk-Tait equation and subsequently, the continuity equation is obtained in the cases of commutativity and noncommutativity, without forgetting that we have investigated the Fisk-Tait equation in
NCST in two cases of potential types. In the first case, we considered a constant magnetic field, but for the second case, we considered a non-constant magnetic field. This, in turn, is responsible for generating a new quantity (for the first case) which is a correction of the density type. We have shown in the present paper that the noncommutativity in space-time affected the continuity equation by causing a new noncommutative quantity of the current density type. However, this effect does not solve the problem of the indefiniteness of the total charge, as expected. This leads us again to think about the difficulties of this type of equations and their problems that are almost endless. Therefore, the noncommutativity in space-time is useless in this regard, contrary to its simplicity and its contribution to solving some problems in the equations that describe the particles of simple spin such as the Dirac equation.

The space-time noncommutativity effect is introduced through the Moyal-Weyl product and under the condition $\Theta=0$, both the Fisk-Tait equation and the continuity equation in NCST return to that of the usual commutative QM. Furthermore, we have shown in this work the linking between the fermions and the bosons in Fock space using the Holstein-Primakoff representation, without forgetting that we found also the spin current density in the two different spin directions.

The results of the present work can be used to expand the study of the motion of the spin- $3 / 2$ particles in the NCST. Finally, we point out that we plan to investigate the reflexion and the transmission coefficients depending on these results, to investigate the Klein paradox.

\section{Acknowledgments}

The author would like to thank Prof. Lyazid Chetouani for his interesting comments and suggestions, and appreciates the unknown referee's valuable and profound comments. 
[1] W. Rarita, J. Schwinger, Phys. Rev. 60, 61 (1941); https://doi.org/10.1103/PhysRev.60.61.

[2] M. A. K. Khalil, M. Seetharaman, Phys. Rev. D 18, 3040 (1978); https://doi.org/10.1103/PhysRevD.18.3040.

[3] J. O. Eeg, Phys. Rev. D 14, 2197 (1976); https://doi. org/10.1103/PhysRevD.14.2197.

[4] H. J. Bhabha, Phil. Mag. 43, 33 (1952).

[5] T. S. Santhanam, A. R. Tekumalla, Fortschr. Phys. 22, 431 (1974); https://doi.org/10.1002/prop. 19740220802.

[6] V. Bargmann, E. P. Wigner, Proc. Natl. Acad. Sci. USA 34, 211 (1948); https://doi.org/10.1073/pnas.34.5. 211.

[7] A. K. Nagpal, J. Phys. A 10, 1803 (1977); https://doi. org/10.1088/0305-4470/10/10/014.

[8] G. B. Mainland, E. C. G. Sudarshan, Phys. Rev. D 8, 1088 (1973); https://doi.org/10.1103/PhysRevD.8. 1088.

[9] V. M. Simulik, Univers. J. Phys. Applicat. 11, 202 (2017); https://doi.org/10.13189/ujpa.2017. 110602.

[10] M. Fierz, W. Pauli, Proc. R. Soc. Lond. A 173, 211 (1939); https://doi.org/10.1098/rspa.1939.0140.

[11] G. Velo, D. Zwanziger, Phys. Rev. 186, 1337 (1969); https://doi.org/10.1103/PhysRev.186.1337.

[12] J. Prabhakaran, M. Seetharaman, P. M. Mathews, J. Phys. A 8, 560 (1975); https://doi.org/10.1088/ 0305-4470/8/4/018.

[13] K. Johnson, E. C. G. Sudarshan, Ann. Phys. 13, 126 (1961); https://doi.org/10.1016/0003-4916(61) 90030-6.

[14] K. K. Gupta, Math. Phys. Sci. A 222118 (1954); https: //doi.org/10.1098/rspa.1954.0057.

[15] T. Fukuyama, K. Yamamoto, Progress Theor. Phys. 49, 1, 304 (1973); https://doi.org/10.1143/PTP.49.304.

[16] V. E. Barnes et al., Phys. Rev. Lett. 12, 204 (1964); https://doi.org/10.1103/PhysRevLett.12.204.

[17] R. Aaij et al., Phys. Rev. Lett. 118, 182001 (2017); https://doi.org/10.1103/PhysRevLett.118.182001.

[18] H. Garcilazo et al., J. Phys. G 34, 961 (2007); https: //doi.org/10.1088/0954-3899/34/5/014.

[19] H. Kim et al., Sci. Adv. 4, eaao4513 (2018); https:// doi.org/10.1126/sciadv . aao4513.

[20] T. Moroi, H. Murayama, M. Yamaguchi, Phys. Lett. B 303, 289 (1993); https://doi.org/10.1016/ 0370-2693 (93) 91434-0.
[21] E. J. Copeland, O. Seto, Phys. Rev. D 72, 023506 (2005); https://doi.org/10.1103/PhysRevD.72.023506.

[22] C. Fisk, W. Tait, J. Phys. A 6, 383 (1973); https:// doi.org/10.1088/0305-4470/6/3/012.

[23] M. Seetharaman et al., Phys. Rev. D 123195 (1975); https://doi.org/10.1103/PhysRevD.12.3195.

[24] R. K. Singh, Indian J. Phys. 93, 503 (2019); https:// doi.org/10.1007/s12648-018-1316-z.

[25] D. Kastler, J. Math. Phys, 41, 3867 (2000); https:// doi.org/10.1063/1.533330.

[26] J. Madore, in Proceedings of Corfu Summer Institute on Elementary Particle Physics - PoS (corfu98) (Sissa Medialab, 1999), art. 016; https://doi.org/10.22323/ 1.001 .0016 .

[27] N. Seiberg, E. Witten, J. Hight Energ. Phys. 1999, 032 (1999); https://doi.org/10.1088/1126-6708/1999/ 09/032.

[28] E. Di Grezia, G. Esposito, A. Funel, G. Mangano, G. Miele, Phys. Rev. D 68, 105012 (2003); https://doi.org/ 10.1103/PhysRevD.68.105012.

[29] O. Bertolami, J. G. Rosa, C. M. L. de Aragro, P. Castorina, D. Zappala Phys. Rev. D 72, 025010 (2005); https: //doi.org/10.1103/PhysRevD.72.025010.

[30] F. Delduc et al., J. Phys. Conf. Ser. 103, 012020 (2008); https://doi.org/10.1088/1742-6596/103/1/012020.

[31] I. Haouam, Symmetry 11, 223 (2019); https://doi. org/10.3390/sym11020223.

[32] I. Haouam, Open J. Microphys. 9, 15 (2019); https: //doi.org/10.4236/ojm.2019.93003.

[33] I. Haouam, L. Chetouani, J. Mod. Phys. 9, 2021 (2018); https://doi.org/10.4236/jmp. 2018.911127.

[34] G. F. Torres Del Castillo, J. Velázquez Castro, Rev. Mex. Fis.50, 306 (2004).

[35] S. Azakov, in Physics of Spin in Solids: Materials, Methods and Applications. NATO Science Series II: Mathematics, Physics and Chemistry. Vol 156, edited by S. Halilov (Springer, Dordrecht, 2004); https://doi. org/10.1007/1-4020-2708-7_7.

[36] C. E. Alonso, J. M. Arias, J. Dukelsky, S. Pittel, Nucl. Phys. A 539, 391 (1992); https://doi.org/10.1016/ 0375-9474 (92) 90283-P.

[37] E. R . Marshalek, Nucl. Phys. A 347, 253 (1980).

[38] J. Dukelsky, S. Pittel, Phys. Lett. B 177, 125 (1986); https://doi.org/10.1016/0370-2693(86)91040-3.

\title{
ПРО РІВНЯННЯ ФІСКА-ТЕЙТА ДЛЯ ФЕРМІОНІВ ЗІ СПІНОМ 3/2, ЩО ВЗАЕМОДІЮТЬ IЗ ЗОВНІШНІМ МАГНІТНИМ ПОЛЕМ У НЕКОМУТАТИВНОМУ ПРОСТОРІ-ЧАСІ
}

\author{
Ільяс Гавам \\ Університет Братів Ментурі, Константина, Алжир
}

\begin{abstract}
У цій праці досліджено рівняння Фіска-Тейта для ферміонів, що взаємодіють із зовнішнім магнітним полем у некомутативному просторі-часі. Введено некомутативність простору та часу методом добутку МоялиВейля. Проаналізовано рівняння неперервності як у комутативному, так і в некомутативному просторі-часі. Також досліджено вплив некомутативності простору-часу на 4-вектор густини струму. Також показано, що повний заряд, отриманий із густини ймовірності, не є визначеним, коли простір некомутативний. Крім того, знайдено густину спінового струму у двох різних напрямках спіну. Також досліджено зв'язок між ферміонами та бозонами у просторі Фока за допомогою перетворення Голстейна-Примакова.
\end{abstract}

